\title{
Pontryagin Maximum Principle for Optimal Control of Variational Inequalities
}

\section{Maïtine Bergounioux}

Département de Mathématiques

UMR 6628 - Université d'Orléans

B.P. 6759 - 45067 Orléans Cedex 2

France

\author{
Housnaa Zidani
}

Laboratoire MIP, UMR 5640

UFR MIG - Université Paul Sabatier

118 route de Narbonne, 31062 Toulouse

France

Abstract. In this paper we investigate optimal control problems governed by variational inequalities. We present a method for deriving optimality conditions in the form of Pontryagin's principle. The main tools used are the Ekeland's variational principle combined with penalization and spike variation techniques.

Keywords: Variational Inequalities, Optimal Control, Pontryagin Principle AMS subject classification. 49J20, 49M29

1. Introduction. The purpose of this paper is to present a method for deriving a Pontryagin type maximum principle as a first order necessary condition of optimal controls for problems governed by variational inequalities. We allow various kinds of constraints to be imposed on the state. To be more precise, we consider the following variational inequality

$$
\begin{gathered}
\left.\frac{\partial y}{\partial t}+A y+f(y)+\partial \varphi(y) \ni u \quad \text { in } Q=\Omega \times\right] 0, T[, \\
y=0 \quad \text { on } \Sigma=\Gamma \times] 0, T[, \\
y(0)=y_{o} \quad \text { in } \Omega .
\end{gathered}
$$

where $\Omega \subset \mathbb{R}^{n}, T>0, u$ is a distributed control, $A$ is a second order elliptic operator and $\frac{\partial y}{\partial t}$ denotes the derivative of $y$ with respect to $t ; \partial \varphi(y)$ is the subdifferential of the function $\varphi$ at $y$. We shall give all the definitions we need in Section 3 and (1.1) will be made precise as well. The control variable $u$ and the state variable $y$ must satisfy constraints of the form

$$
u \in U_{a d}=\left\{u \in L^{p}(Q) \mid u(x, t) \in \mathcal{K}_{U}(x, t) \text { a.e. in } Q\right\} \subset L^{p}(Q),
$$

where $\mathcal{K}_{U}$ is a measurable set-valued mapping from $Q$ with closed values in $\mathcal{P}(\mathbb{R})(\mathcal{P}(\mathbb{R})$ being the set of all subsets of $\mathbb{R}$ ),

$$
\Phi(y) \in \mathcal{C}
$$

with $1<p<\infty, \Phi$ is a $\mathcal{C}^{1}$ mapping from $C(\bar{Q})$ into $C(\bar{Q}), \mathcal{C} \subset C(\bar{Q})$ is a closed convex subset with finite codimension.

The control problem is

$$
\inf \left\{J(y, u) \mid y \in C(\bar{Q}), u \in U_{a d},(y, u) \text { satisfies }(1.1),(1.2)\right\}
$$


where the cost functional is defined by

$$
J(y, u)=\int_{Q} F(x, t, y(x, t), u(x, t)) d x d t+\int_{\Omega} L(x, y(x, T)) d x .
$$

Many authors (as for example Barbu [2], Mignot-Puel [17], Yong [23], Bonnans-Tiba [6], Bonnans-Casas [5] or Bergounioux [3]) have already considered control problems for variational inequalities from the theoretical or numerical point of view. Here we are interested in optimality conditions in the form of Pontryagin's principle. The existence of an optimal solution is assumed a priori. The novelty of this paper is twofold: we obtain the optimality conditions in Pontryagin's form and we think that our hypotheses are not far to be minimal. We essentially ask for the state equation to be well posed and assume differentiability of data with respect to the state. We allow various kinds of constraints to be added on the control $u$ and on the state. However, we restrict the study to the case when $\varphi$ is the indicator function of the closed convex set $K_{o}=\{z \in C(\bar{Q}) \mid z \geq 0\}$ so that the variational inequality (1.1) turns to be the so called obstacle problem.

To get Pontryagin's principle, we use a method based on penalization of state constraints, and Ekeland's principle combined with diffuse perturbations [16, 20]. These techniques have been already used by many authors in the case of optimal control of parabolic or elliptic equations [5, 16, 21]. Some of these techniques have been also used for control problem governed by variational inequalities [5, 23, 4]. In these papers, the variational inequality is approximated via the Moreau-Yosida approximation of the maximal monotone operator $\partial \varphi$.

Here, we use another idea based on the formulation of (1.1) with a slackness variable and the regularity of its solution. In fact, the solution of (1.1) is also a weak solution of:

$$
\frac{\partial y}{\partial t}+A y+f(y)=u+\xi \quad \text { in } Q, \quad y=0 \quad \text { on } \Sigma, \quad y(0)=y_{o} \quad \text { in } \Omega
$$

where $\xi$ is the Lagrange multiplier associated to the variational inequality and is introduced as an additional control variable. Therefore we obtain a problem $(\widetilde{\mathcal{P}})$ equivalent to $(\mathcal{P})$, with constraints on both the control variable and the state variable as well as coupled state/control constraints. We first give a Pontryagin's principle for $(\widetilde{\mathcal{P}})$. For this, we adapt the proof given in $[21,24,7]$ to problem $(\widetilde{\mathcal{P}})$. Next we derive optimality conditions for $(\mathcal{P})$ from those for $(\widetilde{\mathcal{P}})$.

2. Assumptions. Let $\Omega$ be a open, smooth (with a $\mathcal{C}^{2}$ boundary $\Gamma$ for example) and bounded domain of $\mathbb{R}^{n}(2 \leq n)$. In all the sequel, we suppose that

$$
p>n .
$$

REMARK 2.1. We must underline that this choice of $p$ is not optimal. Indeed, we should distinguish the integers $p$ (for the $L^{p}$-space of the distributed control $u$ ) and $q$ (for the $L^{q}$-space of the initial value $\left.y_{o}\right)$. The optimal choice should be $u \in L^{p}(Q)$ with $p>\frac{n}{2}+1$ and $y_{o} \in W_{o}^{1, q}(\Omega)$ with $q>n$; each time it is possible, we will mention how the next assumptions could be weakened from this point of view. Anyhow, to make the presentation clearer we simply assume that $p=q>n$.

We suppose in addition that 
(A1) $A$ is a linear elliptic differential operator defined by

$$
\begin{gathered}
A y=-\sum_{i, j=1}^{n} \partial_{x_{i}}\left(a_{i j}(x) \partial_{x_{j}} y\right)+a_{0}(x) y \text { with } \\
a_{i j} \in \mathcal{C}^{2}(\bar{\Omega}) \text { for } i, j=1 \cdots n, \\
a_{0} \in L^{\infty}(\Omega), \sum_{i, j=1}^{n} a_{i j}(x) \xi_{i} \xi_{j} \geq m_{o} \sum_{i=1}^{n} \xi_{i}^{2}, \forall x \in \bar{\Omega}, \forall \xi \in \mathbb{R}^{\mathrm{n}}, m_{o}>0,
\end{gathered}
$$

(A2) $f: \mathbb{R} \rightarrow \mathbb{R}$ is a monotone increasing, globally lipschitz $\mathcal{C}^{1}$-function.

REMARK 2.2. The monotonicity assumption on $f$ can be relaxed and replaced by:

$$
\exists c_{o} \in \mathbb{R} \quad f_{y}^{\prime} \geq c_{o} .
$$

An appropriate translation shows that we retrieve the case where $f$ is monotonically increasing. So we assume it for the sake of simplicity.

On the other hand one could consider a mapping $f$ from $\mathbb{R} \times \mathbb{R}$ to $\mathbb{R}$ depending on both $y$ and $u$. The method would work in the same way.

(In what follows, we denote the real function $f: \mathbb{R} \rightarrow \mathbb{R}$ and the Nemytski operator associated to $f: y(\cdot) \mapsto f(y(\cdot))$ in $L^{p}(Q)$ by the same symbol $f$.)

(A3) $\varphi: W_{o}^{1, p}(\Omega) \rightarrow \mathbb{R} \cup\{+\infty\}$ is proper (i.e. non identically equal to $+\infty$ ), convex, lower semicontinuous function such that $0 \in \operatorname{dom} \varphi$.

(A4) $y_{o} \in \operatorname{dom} \varphi$.

(A5) For every $(y, u) \in \mathbb{R}^{2}, F(\cdot, y, u)$ is measurable on $Q$. For almost every $(x, t) \in Q$, for every $u \in \mathbb{R}$, $F(x, t, \cdot, u)$ is $\mathcal{C}^{1}$ on $\mathbb{R}$. For almost every $(x, t) \in Q, F(x, t, \cdot)$ and $F_{y}^{\prime}(x, t, \cdot)$ are continuous on $\mathbb{R}^{2}$. The following estimate holds

$$
|F(x, t, y, u)|+\left|F_{y}^{\prime}(x, t, y, u)\right| \leq\left(M_{1}(x, t)+m_{1}|u|^{p}\right) \eta(|y|),
$$

where $M_{1} \in L^{1}(Q), m_{1} \geq 0$ and $\eta$ is a nondecreasing function from $\mathbb{R}^{+}$to $\mathbb{R}^{+}$.

(A6) - For every $y \in \mathbb{R}, L(\cdot, y)$ is measurable on $\Omega$. For almost every $x \in \Omega, L(x, \cdot)$ is $\mathcal{C}^{1}$ on $\mathbb{R}$. The following estimate holds

$$
|L(x, y)|+\left|L_{y}^{\prime}(x, y)\right| \leq M_{2}(x) \eta(|y|),
$$

where $M_{2} \in L^{1}(\Omega), \eta$ is as in (A5).

(A7) $\Phi$ is a $\mathcal{C}^{1}$ mapping from $C(\bar{Q})$ into $C(\bar{Q})$, and $\mathcal{C}$ is a closed convex subset of $C(\bar{Q})$ with finite codimension.

We recall that for $p \in \mathbb{N}$

$$
\begin{gathered}
W^{1, p}(\Omega)=\left\{y \in L^{p}(\Omega) \mid \nabla y \in L^{p}(\Omega)^{n}\right\} \text { and } \\
W^{2,1, p}(Q)=\left\{y \in L^{p}(Q) \mid D y, D^{2} y, \frac{\partial y}{\partial t} \in L^{p}(Q)\right\} .
\end{gathered}
$$

3. Existence and Regularity of Solutions to the Variational Inequality. Let be $V$ and $H$ Hilbert spaces such that $V \subset H \subset V^{\prime}$ with continuous and dense injections. We denote by $(\cdot, \cdot)_{V}$ the $V$-scalar product, $\langle\cdot, \cdot\rangle$ the duality product between $V$ and $V^{\prime}$, and $\|\cdot\|_{V}$ the $V$-norm. We consider a 
linear, continuous $V$-elliptic operator $\mathcal{A}$ from $V$ to $V^{\prime}$ and $\phi$ a convex, proper and lower semi-continuous function from $V$ to $\mathbb{R} \cup\{+\infty\}$. Then we may define the variational inequality

$$
\left\{\begin{array}{c}
\frac{\partial y}{\partial t}(t)+\mathcal{A} y(t)+\partial \phi(y)(t) \ni u(t) \text { a.e. } \mathrm{t} \text { in }[0, T] \\
y(0)=y_{o}
\end{array}\right.
$$

in the following (variational) sense

$$
\left\langle\frac{\partial y}{\partial t}(t)+\mathcal{A} y(t), y(t)-z\right\rangle+\phi(y(t))-\phi(z) \leq\langle f(t), y(t)-z\rangle \quad \text { a.e. } t \in(0, T), \forall z \in V
$$

Here $\partial \phi(y(t))$ denotes the subdifferential of $\phi$ at $z=y(t) \in V$ :

$$
\partial \phi(z)=\left\{z^{*} \in V^{\prime} \mid \phi(z)-\phi(\zeta) \leq\left\langle z-\zeta, z^{*}\right\rangle, \forall \zeta \in V\right\} .
$$

Now, we set $V=H_{o}^{1}(\Omega)$ and $H=L^{2}(\Omega)$; we let $g$ be a primitive function of $f$ (such that $g(0)=0$ for example) and define

$$
\phi=\varphi+g,
$$

where $\varphi$ is given by (A3). Then $\partial \phi=g^{\prime}+\partial \varphi=f+\partial \varphi(g$ is the regular part of $\phi)$. Therefore (1.1) makes sense in the (3.1) form with $\mathcal{A}=A$ and we may give a first existence and regularity result

Theorem 3.1. Set $p \geq 2$; let be $u \in L^{p}(Q)$ and $y_{o} \in W_{o}^{1, p}(\Omega)$. Assume that

$$
\exists \gamma \in L^{p}(\Omega) \cap \partial \varphi\left(y_{o}\right)
$$

then (1.1) has a unique solution $y \in W^{2,1, p}(Q)$.

Proof - We first use a result of Tiba [22], Theorem 4.5 p. 26 that ensures that, if $\beta$ is a maximal monotone graph $\subset \mathbb{R} \times \mathbb{R}, u \in L^{p}(Q)$ and $y_{o} \in W_{o}^{1, p}(\Omega)$, then the following parabolic variational inequality

$$
\left\{\begin{array}{lll}
\frac{\partial y}{\partial t}+A y+\beta(y) & \ni u & \text { a.e. in } Q \\
y(0, x) & =y_{o}(x) & \text { a.e. on } \Omega \\
y(t, x) & =0 & \text { a.e. on } \Sigma
\end{array}\right.
$$

has a unique solution in $W^{2,1, p}(Q)$ if the compatibility relation

$$
0 \in \operatorname{dom} \beta, y_{o}(x) \in \operatorname{dom} \beta \text { a.e. in } \Omega,
$$

$$
\exists \gamma \in L^{p}(\Omega) \text { such that } \gamma(x) \in \beta\left(y_{o}(x)\right) \text { a.e. in } \Omega \text {, }
$$

is fulfilled. One can apply this result to $\beta=f+\partial \varphi$ which is a maximal monotone graph since $f$ is monotone increasing and $\varphi$ is convex, lower semicontinuous and proper. It remains to check (3.6) that is

$$
\exists \gamma \in L^{p}(\Omega) \text { such that } \gamma(x) \in f\left(y_{o}(x)\right)+\partial \varphi\left(y_{o}(x)\right) \text { a.e. in } \Omega \text {. }
$$

This is equivalent to

$$
\exists \gamma \text { such that } \gamma+f\left(y_{o}\right) \in L^{p}(\Omega) \text {, and } \gamma(x) \in \partial \varphi\left(y_{o}(x)\right) \text { a.e. in } \Omega \text {. }
$$

As $f$ is globally lipschitz then $f\left(y_{o}\right) \in L^{p}(\Omega)$ and we get the result 
We set

$$
\xi=u-\frac{\partial y}{\partial t}-A y-f(y) \in L^{p}(Q)
$$

(since $f$ is globally lipschitz and $y \in W^{2,1, p}(Q)$ ). In addition, $\xi(t) \in \partial \varphi(y(t))$ almost everywhere in $] 0, T[$; using the characterization of the subdifferential of a function in Banach spaces this gives

$$
\left.\varphi(y(t))+\varphi^{*}(\xi(t))-\langle y(t), \xi(t)\rangle=0 \text { a.e in }\right] 0, T[.
$$

In this last relation $\langle$,$\rangle denotes the duality product between V=W_{o}^{1, p}(\Omega)$ and $V^{\prime}$, and $\varphi^{*}$ is the conjugate function of $\varphi$. For more details one can refer to Barbu and Precupanu [1] or Ekeland-Temam [13]. It follows that the variational inequality (1.1) is equivalent to

$$
\begin{aligned}
\frac{\partial y}{\partial t}+A y+f(y) & =u+\xi & & \text { in } Q, \\
y & =0 & & \text { on } \Sigma, \\
y(x, 0) & =y_{o}(x) & & \text { in } \Omega,
\end{aligned}
$$

and (3.7). As $y_{o} \in W_{o}^{1, p}(\Omega)$, and $(u, \xi) \in L^{p}(Q) \times L^{p}(Q)$, the solution $y$ of equation (3.8) belongs to $C(\bar{Q}) \cap W^{2,1, p}(Q)$. More precisely, we have:

THEOREM 3.2. (i) If $p>\frac{n}{2}+1$ and $\left(u, \xi, y_{o}\right) \in L^{p}(Q) \times L^{p}(Q) \times \mathcal{C}(\bar{\Omega})$ then equation (3.8) has a unique weak solution $y_{u \xi}$ in $W(0, T) \cap C(\bar{Q})$ which satisfies

$$
\left\|y_{u \xi}\right\|_{\infty, Q} \leq C_{1}\left(\|u\|_{p, Q}+\|\xi\|_{p, Q}+\left\|y_{o}\right\|_{\infty, \Omega}+1\right),
$$

where $C_{1}=C_{1}\left(T, \Omega, m_{0}, n, p\right)$. Moreover, for every $\varepsilon>0, y_{u \xi}$ is Hölder continuous on $[\varepsilon, T] \times \bar{\Omega}$ and belongs to $W^{2,1, p}(\Omega \times] \varepsilon, T[)$.

(ii) if $p>n$ and $\left(u, \xi, y_{o}\right) \in L^{p}(Q) \times L^{p}(Q) \times W_{o}^{1, p}(\Omega)$, equation (3.8) has a unique weak solution $y_{u \xi}$ in $W^{2,1, p}(Q) \cap C(\bar{Q})$.

Proof - The existence of a unique weak solution $y_{u \xi}$ in $W(0, T) \cap C(\bar{Q})$ for equation (3.8), can be proved as in the case of Robin boundary condition (see Raymond-Zidani [20, 21]). The Hölder continuity result holds thanks to [9].

The point (ii) can be found in Bergounioux-Tröltzsch [4].

\section{Optimal Control of the Obstacle Problem.}

4.1. The Obstacle problem. Now we focus on the very case of control of obstacle problem where

$$
K_{o}=\left\{z \in W_{o}^{1, p}(\Omega) \mid z \geq 0 \text { a.e. in } \Omega\right\},
$$

and $\varphi$ is the indicator function of $K_{o}$ :

$$
\varphi(z)=\left\{\begin{array}{cc}
0 & \text { if } z \in K_{o} \\
+\infty & \text { else }
\end{array}\right.
$$

It is clear that $0 \in \operatorname{dom} \varphi=K_{o}$. Moreover the compatibility condition (3.5) is fulfilled with $\gamma=0$, so that Theorem 3.1 is valid. On the other hand, the (classical) calculus of $\varphi^{*}$ show that relation (3.7) is equivalent to

$$
y(t) \geq 0 \text { in } \Omega \quad \forall t \in] 0, T\left[\quad, \quad \xi(t) \geq 0 \text { in } \Omega \text { and } \int_{\Omega} y(t, x) \xi(t, x) d x=0 \text { a.e. } t \in\right] 0, T[,
$$


that is, at last

$$
y \geq 0 \text { in } Q \quad, \xi \geq 0 \text { a.e. in } Q \text { and } \int_{Q} y(t, x) \xi(t, x) d x d t=0
$$

We may summarize in the following

TheOrem 4.1. Assume $p>n,\left(u, y_{o}\right) \in L^{p}(Q) \times W_{o}^{1, p}(\Omega)$; then the following variational vnequality

$$
\frac{\partial y}{\partial t}+A y+f(y)+\partial \varphi(y) \ni u \quad \text { in } Q, \quad y=0 \quad \text { on } \Sigma, \quad y(0)=y_{o} \quad \text { in } \Omega
$$

where $\varphi$ is the indicator function of $K_{o}$, has a unique solution $y \in \mathcal{C}(\bar{Q}) \cap W^{2,1, p}(Q)$. Moreover it is equivalent to

$$
\left\{\begin{aligned}
\frac{\partial y}{\partial t}+A y+f(y)=u+\xi & \text { in } Q, \quad y=0 \quad \text { on } \Sigma, \quad y(x, 0)=y_{o}(x) \quad \text { in } \Omega, \\
\xi \geq 0, \quad y \geq 0, & \int_{Q} y(t, x) \xi(t, x) d x d t=0 .
\end{aligned}\right.
$$

In the sequel we denote

$$
V_{a d}=\left\{\xi \in L^{p}(Q) \mid \xi \geq 0 \text { a.e. in } Q\right\}
$$

4.2. Pontryagin principle . From now we consider the following problem $(\widetilde{\mathcal{P}})$ :

Minimize $J(y, u)$ subject to :

$$
\begin{array}{ll}
\frac{\partial y}{\partial t}+A y+f(y)=u+\xi \text { in } Q, & y=0 \text { on } \Sigma, y(., 0)=y_{o} \text { in } \Omega \\
\tilde{\Phi}(y) \in \widetilde{\mathcal{C}} & \text { ("Pure" state constraint) } \\
(u, \xi) \in U_{a d} \times V_{a d} & \text { ("Pure" control constraints) } \\
\int_{Q} y(t, x) \xi(t, x) d x d t=0 & \text { (Mixed State/Control integral constraints) }
\end{array}
$$

where

$$
\tilde{\Phi}(y)=(\Phi(y), y) \text { and } \widetilde{\mathcal{C}}=\mathcal{C} \times\{y \in \mathcal{C}(\bar{Q}) \mid y \geq 0\}
$$

The results of Section 3. yield that problems $(\mathcal{P})$ and $(\widetilde{\mathcal{P}})$ are equivalent. In particular, if $(\bar{y}, \bar{u})$ is solution of $(\mathcal{P})$, then there exists $\bar{\xi} \in L^{p}(Q)$ such that $(\bar{y}, \bar{u}, \bar{\xi})$ is an optimal solution of $(\widetilde{\mathcal{P}})$ with $\bar{\xi}=\frac{\partial \bar{y}}{\partial t}+A \bar{y}+f(\bar{y})-\bar{u}$. Let us mention that we are not interested in existence results (though we will give an example in the last section of this paper ) but in optimality conditions for $(\bar{y}, \bar{u})$. Consequently, we study optimality conditions for $(\bar{y}, \bar{u}, \bar{\xi})$ to get those for $(\bar{y}, \bar{u})$.

Let us define the Hamiltonian functions by:

$$
H_{1}(x, t, y, u, q, \nu)=\nu F(x, t, y, u)+q u
$$

for every $(x, t, y, u, q, \nu) \in Q \times \mathbb{R}^{4}$, and

$$
H_{2}(y, \xi, q, \lambda)=q \xi+\lambda y \xi
$$


for every $(y, \xi, q, \lambda) \in \mathbb{R}^{4}$.

THEOREM 4.2. [Pontryagin Principle for $(\widetilde{\mathcal{P}})$ ] If $(A 1)-(A 7)$ are fulfilled and if $(\bar{y}, \bar{u}, \bar{\xi})$ is a solution of $(\widetilde{\mathcal{P}})$, then there exist $\bar{q} \in L^{1}\left(0, T ; W_{o}^{1,1}(\Omega)\right), \bar{\nu} \in \mathbb{R}, \bar{\lambda} \in \mathbb{R}$ and $(\bar{\mu}, \bar{\theta}) \in \mathcal{M}(\bar{Q}) \times \mathcal{M}(\bar{Q})(\mathcal{M}(\bar{Q})$ is the space of Radon measures on $\bar{Q})$ ), such that

$$
\begin{gathered}
(\bar{\nu}, \bar{\lambda}, \bar{\mu}, \bar{\theta}) \neq 0, \quad \bar{\nu} \geq 0, \\
\forall z \in\{z \in \mathcal{C}(\bar{Q}) \mid z \geq 0\} \quad\langle\bar{\mu}, z-\bar{y}\rangle_{\bar{Q}} \leq 0, \quad \text { and } \forall z \in \mathcal{C} \quad\langle\bar{\theta}, z-\Phi(\bar{y})\rangle_{\bar{Q}} \leq 0 \\
\left\{\begin{array}{cc}
-\frac{\partial \bar{q}}{\partial t}+A^{*} \bar{q}+f_{y}^{\prime}(\bar{y}) \bar{q}=\bar{\nu} F_{y}^{\prime}(x, t, \bar{y}, \bar{u})+\left.\bar{\mu}\right|_{Q}+\left.\left[\Phi^{\prime}(\bar{y})^{*} \bar{\theta}\right]\right|_{Q}+\bar{\lambda} \bar{\xi} & \text { in } Q, \\
\bar{q}=0 \quad \text { on } \Sigma, \quad \bar{q}(T)=\bar{\nu} L_{y}^{\prime}(x, \bar{y}(T))+\left.\bar{\mu}\right|_{\bar{\Omega}_{T}}+\left.\left[\Phi^{\prime}(\bar{y})^{*} \bar{\theta}\right]\right|_{\bar{\Omega}_{T}} & \text { in } \Omega,
\end{array}\right.
\end{gathered}
$$$$
\bar{q} \in L^{\delta^{\prime}}\left(0, T ; W_{o}^{1, d^{\prime}}(\Omega)\right) \quad \text { for every }(\delta, d) \text { satisfying } \frac{n}{2 d}+\frac{1}{\delta}<\frac{1}{2},
$$

where $\left.\bar{\mu}\right|_{Q}\left(\right.$ resp. $\left.\left.\left[\Phi^{\prime}(\bar{y})^{*} \bar{\theta}\right]\right|_{Q}\right)$ is the restriction of $\bar{\mu}\left(\right.$ resp. $\left.\left[\Phi^{\prime}(\bar{y})^{*} \bar{\theta}\right]\right)$ to $Q$, and $\left.\bar{\mu}\right|_{\bar{\Omega}_{T}}\left(\right.$ resp. $\left.\left.\left[\Phi^{\prime}(\bar{y})^{*} \bar{\theta}\right]\right|_{\bar{\Omega}_{T}}\right)$ is the restriction of $\bar{\mu}\left(\right.$ resp. $\left.\left[\Phi^{\prime}(\bar{y})^{*} \bar{\theta}\right]\right)$ to $\bar{\Omega} \times\{T\},\langle\cdot, \cdot\rangle_{\bar{Q}}$ denotes the duality product between $\mathcal{M}(\bar{Q})$ and $C(\bar{Q}), A^{*}$ is the adjoint operator of $A$ and $\frac{1}{d}+\frac{1}{d^{\prime}}=1$.

REMARK 4.1. Let us comment these relations for a while: $(\bar{\mu}, \bar{\theta})$ are the multipliers associated to the state constraints; $\bar{\mu}$ corresponds to " $y \geq 0$ " and an immediate consequence of relation (4.10b) is the following complementarity result: $\bar{\mu} \leq 0,\langle\bar{\mu}, \bar{y}\rangle_{\bar{Q}}=0 . \bar{\theta}$ is associated to the (general) constraint " $\Phi(y) \in \mathcal{C} "$.

$\bar{\lambda}$ is the multiplier associated to the integral constraint " $\int_{Q} y(t, x) \xi(t, x) d x d t=0$ " and $\bar{q}$ is the classical adjoint state which takes into account the cost functional via $\bar{\nu}$.

Condition (4.10a) is a non triviality condition. We must underline that we get (a priori) non qualified optimality conditions. If $\bar{\nu} \neq 0$, the problem turns to be qualified.

REMARK 4.2. One may note that if $\bar{\xi}=0$, then it could happen that $\bar{\nu}=\bar{\mu}=\bar{\theta}=0$ and $\bar{\lambda} \neq 0$, so that $\bar{q}=0$; therefore, the optimality system could appear as completely unuseful. However, this is the case where the solution $(\bar{y}, \bar{u})$ is solution of a control problem governed by a classical semilinear parabolic equation since we have $\frac{\partial \bar{y}}{\partial t}+A \bar{y}+f(\bar{y})=\bar{u}$, and the associated optimality systems are well known for this kind of problems. We refer for instance to [20].

Theorem 4.3. [Pontryagin Principle for $(\mathcal{P})$ ] If $(A 1)-(A 7)$ are fulfilled and if $(\bar{y}, \bar{u})$ is a solution of $(\mathcal{P})$, then there exists $\bar{q} \in L^{1}\left(0, T ; W_{o}^{1,1}(\Omega)\right), \bar{\nu} \in \mathbb{R}, \bar{\lambda} \in \mathbb{R},(\bar{\mu}, \bar{\theta}) \in \mathcal{M}(\bar{Q}) \times \mathcal{M}(\bar{Q})$ such that (4.10a), (4.10b), (4.10d), and (4.10e) hold. Moreover, we have

(4.11a) $\left\{\begin{array}{c}-\frac{\partial \bar{q}}{\partial t}+A^{*} \bar{q}+f_{y}^{\prime}(\bar{y}) \bar{q}=\bar{\nu} F_{y}^{\prime}(x, t, \bar{y}, \bar{u})+\bar{\mu}_{\left.\right|_{Q}}+\left[\Phi^{\prime}(\bar{y})^{*} \bar{\theta}\right]_{\left.\right|_{Q}}+\bar{\lambda}\left(\frac{\partial \bar{y}}{\partial t}+A \bar{y}+f(\bar{y})-\bar{u}\right) \text { in } Q, \\ \bar{q}=0 \quad \text { on } \Sigma, \quad \bar{q}(T)=\bar{\nu} L_{y}^{\prime}(x, \bar{y}(T))+\left.\bar{\mu}\right|_{\bar{\Omega}_{T}}+\left.\left[\Phi^{\prime}(\bar{y})^{*} \bar{\theta}\right]\right|_{\bar{\Omega}_{T}} \quad \text { in } \Omega,\end{array}\right.$ 


$$
\bar{q}(x, t)\left(\frac{\partial \bar{y}}{\partial t}+A \bar{y}+f(\bar{y})-\bar{u}\right)(x, t)=0 \quad \text { a.e. }(x, t) \in Q .
$$

REMARK 4.3. Relation (4.11b) is a pointwise complementarity condition. Therefore, $\bar{q}$ may be viewed as a Lagrange multiplier associated to the pointwise constraint " $\left.\frac{\partial y}{\partial t}+A y+f(y)-u\right)(x, t) \geq 0$ ".

Let us recall a regularity result for weak solution of parabolic equation with measures as data:

Proposition 4.1. Let $\mu$ be in $\mathcal{M}_{b}(\bar{Q} \backslash(\bar{\Omega} \times\{0\} \cup \bar{\Sigma}))$ and let a be in $L^{p}(Q)$ satisfying

$$
a \geq C_{0}, \quad\|a\|_{L^{p}(Q)} \leq M
$$

where $M>0$. Consider the equation

$$
-\frac{\partial q}{\partial t}+A^{*} q+a q=\mu_{Q} \quad \text { in } Q, \quad q=0 \quad \text { on } \Sigma, \quad q(T)=\mu_{\bar{\Omega}_{T}} \quad \text { on } \bar{\Omega},
$$

where $\mu=\mu_{Q}+\mu_{\bar{\Omega}_{T}}$ is a bounded Radon measure on $\bar{Q} \backslash(\bar{\Omega} \times\{0\} \cup \bar{\Sigma})$, $\mu_{Q}$ is the restriction of $\mu$ to $Q$, and $\mu_{\bar{\Omega}_{T}}$ is the restriction of $\mu$ to $\bar{\Omega} \times\{T\}$. Equation (4.12) admits a unique weak solution $q \in L^{1}\left(0, T ; W_{o}^{1,1}(\Omega)\right)$. For every $(\delta, d)$ satisfying $d>2, \delta>2, \frac{n}{2 d}+\frac{1}{\delta}<\frac{1}{2}, q \in L^{\delta^{\prime}}\left(0, T ; W_{o}^{1, d^{\prime}}(\Omega)\right)$ and we have:

$$
\|q\|_{L^{\delta^{\prime}}\left(0, T ; W_{o}^{1, d^{\prime}}(\Omega)\right)} \leq C_{2}\|\mu\|_{\mathcal{M}_{b}(\bar{Q} \backslash(\bar{\Omega} \times\{0\} \cup \bar{\Sigma}))},
$$

where $C_{2}=C_{2}\left(T, \Omega, n, C_{0}, M, p, \delta, d\right)$ is independent of $a$. Moreover, there exists a function $q(0) \in L^{1}(\Omega)$ such that:

$$
\int_{Q} q\left\{\frac{\partial y}{\partial t}+A y+a y\right\} d x d t=\langle y, \mu\rangle_{b}-\langle y(0), q(0)\rangle_{C(\bar{\Omega}) \times \mathcal{M}(\bar{\Omega})}
$$

for every $y \in Y=\left\{y \in W(0, T) \cap C(\bar{Q}) \mid \frac{\partial y}{\partial t}+A y \in L^{p}(Q), y=0\right.$ in $\left.\left.\Sigma\right)\right\}$, where $\langle\cdot, \cdot\rangle_{b}$ denotes the duality product between $C_{b}(\bar{Q} \backslash(\bar{\Omega} \times\{0\} \cup \bar{\Sigma}))$ and $\mathcal{M}_{b}(\bar{Q} \backslash(\bar{\Omega} \times\{0\} \cup \bar{\Sigma})) .\left(C_{b}(\bar{Q} \backslash(\bar{\Omega} \times\{0\} \cup \bar{\Sigma}))\right.$ denotes the space of bounded continuous functions on $\bar{Q} \backslash(\bar{\Omega} \times\{0\} \cup \bar{\Sigma})$, while $\mathcal{M}_{b}(\bar{Q} \backslash(\bar{\Omega} \times\{0\} \cup \bar{\Sigma}))$ denotes the space of bounded Radon measures on $\bar{Q} \backslash(\bar{\Omega} \times\{0\} \cup \bar{\Sigma})$, that is, the topological dual of $C_{o}(\bar{Q} \backslash(\bar{\Omega} \times\{0\} \cup \bar{\Sigma}))$.) Proof - The proof is the same to the one given in [19] for the Neumann boundary conditions (see also [7]). An easy adaptation of this proof yields the previous result. However, for the convenience of the reader we recall that $q$ is the weak solution of (4.12) if and only if $q$ belongs to $L^{1}\left(0, T ; W_{o}^{1,1}(\Omega)\right), a q \in L^{1}(Q)$ and for every $\varphi \in C^{1}(\bar{Q})$ satisfying $\varphi(x, 0)=0$ on $\bar{\Omega}$ and $\varphi(\cdot)=0$ on $\Sigma$, we have

$$
\int_{Q}\left\{q \frac{\partial \varphi}{\partial t}+\Sigma_{i, j} a_{i j} D_{j} \varphi D_{i} q+a \varphi q\right\} d x d t=\langle\varphi, \mu\rangle_{b} .
$$

As in [7], we can prove that the weak solution $q$ belongs to $L^{\delta^{\prime}}\left(0, T ; W_{o}^{1, d^{\prime}}(\Omega)\right)$ for every $(\delta, d)$ satisfying the condition

$$
d>2, \quad \delta>2, \quad \frac{n}{2 d}+\frac{1}{\delta}<\frac{1}{2} .
$$

We remark that the set of pairs $(\delta, d)$ satisfying the above condition is nonempty. We remark also that if $(\delta, d)$ satisfies (4.13), if $a$ belongs to $L^{p}(Q)$ and if $q$ belongs to $L^{\delta^{\prime}}\left(0, T ; W_{o}^{1, d^{\prime}}(\Omega)\right)$, then $a q \in L^{1}(Q)$. Now, since $q \in L^{\delta^{\prime}}\left(0, T ; W_{o}^{1, d^{\prime}}(\Omega)\right)$ (where $(\delta, d)$ satisfies (4.24)), and since

$$
\operatorname{div}_{x t}\left(\left(\Sigma_{j} a_{i j} D_{j} q\right)_{1 \leq i \leq n}, q\right)=\frac{\partial q}{\partial t}-A q \text { belongs to } \mathcal{M}_{b}(Q),
$$


then we can define the normal trace of the vector field $\left(\left(\sum_{j} a_{i j} D_{j} q\right)_{1 \leq i \leq n}, q\right)$, in the space $W^{\frac{-1}{m}, m}(\partial Q)$ (for some $\left.1<m<\frac{n+1}{n}\right)$. If we denote by $\gamma_{o}\left(\left(\sum_{j} a_{i j} D_{j} q\right)_{1 \leq i \leq n}, q\right)$ this normal trace, we can prove (see Theorem 4.2 in [19] that this normal trace belongs to $\mathcal{M}(\partial Q)$ and the restriction of $\gamma_{o}\left(\left(\sum_{j} a_{i j} D_{j} q\right)_{1 \leq i \leq n}, q\right)$ to $\bar{\Omega} \times\{T\}$ is equal to $\mu_{\bar{\Omega}_{T}}$, and if $q(0)$ is the measure on $\bar{\Omega}$ which satisfies the Green formula of Theorem 3.2 , then $-q(0)$ is the restriction of $\gamma_{o}\left(\left(\sum_{j} a_{i j} D_{j} q\right)_{1 \leq i \leq n}, q\right)$ to $\bar{\Omega} \times\{0\}$. In fact, it can be proved that $q(0)$ belongs to $L^{1}(\Omega)$ (see Theorem 4.3 in [19]).

4.3. Proof of Theorems 4.2-4.3. First we assume that Theorem 4.2 is valid. As mentioned before, if $(\bar{y}, \bar{u})$ is an optimal solution for $(\mathcal{P})$, then $(\bar{y}, \bar{u}, \bar{\xi})$ is a solution for $(\widetilde{\mathcal{P}})$, where $\bar{\xi}=\frac{\partial \bar{y}}{\partial t}+A \bar{y}+f(\bar{y})-\bar{u} \in$ $L^{p}(Q)$. Thanks to Theorem 4.2, there exist $(\bar{\nu}, \bar{\lambda}, \bar{\mu}, \bar{q})$ such that (4.10) holds. Replacing $\bar{\xi}$ by its value in (4.10c) obviously leads to (4.11a). Furthermore, relation (4.10f) implies

$$
(\bar{q}(x, t)+\bar{\lambda} \bar{y}(x, t))(\bar{\xi}(x, t)-\xi) \leq 0 \quad \text { a.e. }(x, t) \in Q, \forall \xi \in \mathbb{R}^{+},
$$

which gives

$$
(\bar{q}(x, t)+\bar{\lambda} \bar{y}(x, t)) \bar{\xi}(x, t)=0 \quad \text { a.e. }(x, t) \in Q .
$$

As $\bar{y}(x, t) \bar{\xi}(x, t)=0$ a.e. in $Q$ we obtain (4.11b). This concludes the proof of Theorem 4.3.

It remains to show that Theorem 4.2 is valid. Note that Pontryagin's principle for control problem with unbounded controls, with pointwise state constraints, and with state/control constraints in integral form have been already studied in [7]. For convenience of the reader, we give the main ideas of the proof.

Step 1: Metric space of controls.

In the sequel, we shall consider control problems for which the state constraints (4.6b) and the state/control integral constraints (4.6d) are penalized. These problems are constructed in such a way to make $(\bar{y}, \bar{u}, \bar{\xi})$ be an approximate solution. The idea is to apply next the Ekeland variational principle. For this we have to define a metric space of controls, endowed with the so-called Ekeland distance $d$, to make the mapping $(u, \xi) \longmapsto y_{u \xi}$ continuous from this metric space into $\mathcal{C}(\bar{Q})$. Thanks to Theorem 3.2, this continuity condition will be realized if convergence in the metric space of controls implies convergence in $L^{p}(Q) \times L^{p}(Q)$. Here, since we deal with (generally) unbounded controls, the convergence in $\left(U_{a d} \times V_{a d}, d\right)$ does not imply the convergence in $L^{p}(Q) \times L^{p}(Q)$ (see [14], p. 227). To overcome this difficulty, as in $[24,20]$, we define a new metric as following. For $0<k<\infty$, we set:

$$
\begin{aligned}
& U_{a d}(\bar{u}, k)=\left\{u \in U_{a d}|| u(x, t)-\bar{u}(x, t) \mid \leq k \quad \text { a.e. }(x, t) \in Q\right\} \\
& V_{a d}(\bar{\xi}, k)=\left\{\xi \in V_{a d}|| \xi(x, t)-\bar{\xi}(x, t) \mid \leq k \quad \text { a.e. }(x, t) \in Q\right\} .
\end{aligned}
$$

We endow the control space with Ekeland's metric:

$$
d\left(\left(u_{1}, \xi_{1}\right),\left(u_{2}, \xi_{2}\right)\right)=\mathcal{L}^{n+1}\left(\left\{\left(x, t \mid u_{1}(x, t) \neq u_{2}(x, t)\right\}\right)+\mathcal{L}^{n+1}\left(\left\{(x, t) \mid \xi_{1}(x, t) \neq \xi_{2}(x, t)\right\}\right)\right.
$$

where $\mathcal{L}^{n+1}$ denotes the Lebesgue measure in $\mathbb{R}^{n+1}$. Then as in $[24,20]$, we can prove the following Lemma:

Lemma 4.1. $\left(U_{a d}(\bar{u}, k) \times V_{a d}(\bar{\xi}, k), d\right)$ is a complete metric space for the distance $d$, and the mapping which associates $\left(y_{u \xi}, J\left(y_{u \xi}, u\right)\right)$ with $(u, \xi)$ is continuous from $\left(U_{a d}(\bar{u}, k) \times V_{a d}(\bar{\xi}, k), d\right)$ into $\mathcal{C}(\bar{Q}) \times \mathbb{R}$. 
In [7], the authors have used another method to build the metric space of controls. This construction was adapted to the type of constraints they have considered.

\section{Step 2: $\quad$ Penalized problems}

Since $\mathcal{C}(\bar{Q})$ is separable, there exists a norm $|\cdot|_{\mathcal{C}(\bar{Q})}$, which is equivalent to the norm $\|\cdot\|_{\mathcal{C}(\bar{Q})}$ such that $\left(\mathcal{C}(\bar{Q}),|\cdot|_{\mathcal{C}(\bar{Q})}\right)$ is strictly convex and $\mathcal{M}(\bar{Q})$, endowed with the dual norm of $|\cdot|_{\mathcal{C}(\bar{Q})}\left(\right.$ denoted by $\left.|\cdot|_{\mathcal{M}(\bar{Q})}\right)$, is also strictly convex (see [11], Corollary 2 p. 148 , or Corollary 2 p. 167). Let $\mathbb{K}$ be a convex subset of $\mathcal{C}(\bar{Q})$. We define the distance function to $\mathbb{K}$ (for the new norm $|\cdot|_{\mathcal{C}(\bar{Q})}$ ) by

$$
\delta_{\mathbb{K}}(\zeta)=\inf _{z \in \mathbb{K}}|\zeta-z|_{\mathcal{C}(\bar{Q})} .
$$

Since $\mathbb{K}$ is convex, then $\delta_{\mathbb{K}}$ is convex and Lipschitz of rank 1 , and we have

$$
\underset{\substack{\rho \backslash 0, \zeta^{\prime} \rightarrow \zeta}}{\limsup } \frac{\delta_{\mathbb{K}}\left(\zeta^{\prime}+\rho z\right)-\delta_{\mathbb{K}}\left(\zeta^{\prime}\right)}{\rho}=\max \left\{\langle\xi, z\rangle_{\bar{Q}} \mid \xi \in \partial \delta_{\mathbb{K}}(\zeta)\right\}
$$

for every $\zeta, z \in \mathcal{C}(\bar{Q})$, where $\partial \delta_{\mathbb{K}}(\zeta)$ is the subdifferential of $\delta_{\mathbb{K}}$ at $(\zeta)$. Moreover, as $\mathbb{K}$ is a closed convex subset of $\mathcal{C}(\bar{Q})$, it is proved in ([16], Lemma 3.4) that for every $\zeta \notin \mathbb{K}$, and every $\xi \in \partial \delta_{\mathbb{K}}(\zeta),|\xi|_{\mathcal{M}(\bar{Q})}=1$. Since $\partial \delta_{\mathbb{K}}(\zeta)$ is convex in $\mathcal{M}(\bar{Q})$ and $\left(\mathcal{M}(\bar{Q}),|\cdot|_{\mathcal{M}(\bar{Q})}\right)$ is strictly convex, then if $\zeta \notin \mathbb{K}, \partial \delta_{\mathbb{K}}(\zeta)$ is a singleton and $\delta_{\mathbb{K}}$ is Gâteaux-differentiable at $\zeta$. Let us notice that when $\mathbb{K}:=\{z \in \mathcal{C}(\bar{Q}) \mid z \geq 0\}$, the distance function to $\mathbb{K}$ is given by $\delta_{\mathbb{K}}(\zeta)=\left|\zeta^{-}\right|_{\mathcal{C}(\bar{Q})}$, where $\zeta^{-}=\min (0, \zeta)$.

Endowing $\mathcal{C}(\bar{Q}) \times \mathcal{C}(\bar{Q})$ with the product norm we have similarly $\delta_{\widetilde{\mathcal{C}}}(\tilde{\Phi}(y))^{2}=\left|y^{-}\right|_{\mathcal{C}(\bar{Q})}^{2}+\delta_{\mathcal{C}}(\Phi(y))^{2}(\widetilde{\mathcal{C}}$ is defined by (4.7)). Let us consider the penalized functional:

$$
J_{\varepsilon}(y, u, \xi)=\left\{\left[\left(J(y, u)-J(\bar{y}, \bar{u})+\varepsilon^{2}\right)^{+}\right]^{2}+\delta_{\tilde{\mathcal{C}}}(\tilde{\Phi}(y))^{2}+\left(\int_{Q} y(x, t) \xi(x, t) d x d t\right)^{2}\right\}^{\frac{1}{2}} .
$$

With such a choice, for every $\varepsilon>0$ and $k>0,(\bar{y}, \bar{u}, \bar{\xi})$ is a $\varepsilon^{2}$-solution of the penalized problem

$$
\left(\mathcal{P}_{k, \varepsilon}\right) \quad \inf \left\{J_{\varepsilon}(y, u, \xi) \mid y \in \mathcal{C}(\bar{Q}),(u, \xi) \in U_{a d}(\bar{u}, k) \times V_{a d}(\bar{\xi}, k), \quad(y, u, \xi) \text { satisfies }(4.6 a)\right\},
$$

i.e.

$$
\inf \left(\mathcal{P}_{k, \varepsilon}\right) \leq J_{\varepsilon}(\bar{y}, \bar{u}, \bar{\xi}) \leq \inf \left(\mathcal{P}_{k, \varepsilon}\right)+\varepsilon^{2}
$$

( since $\inf \left(\mathcal{P}_{k, \varepsilon}\right) \geq 0$ and $\left.J_{\varepsilon}(\bar{y}, \bar{u}, \bar{\xi})=\varepsilon^{2}\right)$.

For every $k>0$, we choose $\varepsilon(k)=\varepsilon_{k} \leq \frac{1}{k^{2 p}}$ and we denote by $\left(\mathcal{P}_{k}\right)$ the penalized problem $\left(\mathcal{P}_{k, \varepsilon_{k}}\right)$. Thanks to Ekeland's principle ([13] p. 30), for every $k \geq 1$, there exists $\left(u_{k}, \xi_{k}\right) \in U_{a d}(\bar{u}, k) \times V_{a d}(\bar{\xi}, k)$ such that

$$
\begin{gathered}
d\left(\left(u_{k}, \xi_{k}\right),(\bar{u}, \bar{\xi})\right) \leq \varepsilon_{k} \leq \frac{1}{k^{2 p}}, \\
J_{\varepsilon_{k}}\left(y_{k}, u_{k}, \xi_{k}\right) \leq J_{\varepsilon_{k}}\left(y_{u \xi}, u, \xi\right)+\varepsilon_{k} d\left(\left(u_{k}, \xi_{k}\right),(u, \xi)\right)
\end{gathered}
$$

for every $\left.(u, \xi) \in U_{a d}(\bar{u}, k) \times V_{a d}(\bar{\xi}, k)\right)\left(y_{k}\right.$ and $y_{u \xi}$ being the states corresponding respectively to $\left(u_{k}, \xi_{k}\right)$ and to $(u, \xi))$. In view of the definition of $\varepsilon_{k}$, we have $\lim _{k}\left\|u_{k}-\bar{u}\right\|_{p, Q}=\lim _{k}\left\|\xi_{k}-\bar{\xi}\right\|_{p, Q}=0$. Indeed, $\mathcal{L}^{n+1}\left(\left\{(x, t) \mid u_{k}(x, t) \neq \bar{u}(x, t)\right\}\right)+\mathcal{L}^{n+1}\left(\left\{(x, t) \mid \xi_{k}(x, t) \neq \bar{\xi}(x, t)\right\}\right) \leq \frac{1}{k^{2 p}}$, and $\left|u_{k}(x, t)-\bar{u}(x, t)\right| \leq$ $k,\left|\xi_{k}(x, t)-\bar{\xi}(x, t)\right| \leq k$ a.e. on $Q$. Thus $\left\|u_{k}-\bar{u}\right\|_{p, Q} \leq \frac{1}{k},\left\|\xi_{k}-\bar{\xi}\right\|_{p, Q} \leq \frac{1}{k}$. 
To exploit the approximate optimality conditions (4.15), we introduce a particular perturbation of $\left(u_{k}, \xi_{k}\right)$.

Step 3: Diffuse perturbations.

For fixed $\left(u_{o}, \xi_{o}\right)$ in $U_{a d} \times V_{a d}$, we denote by $\left(u_{o k}, \xi_{o k}\right)(k>0)$ the pair of functions in $U_{a d}(\bar{u}, k) \times V_{a d}(\bar{\xi}, k)$ defined by

$$
\begin{aligned}
& u_{o k}(x, t)= \begin{cases}u_{o}(x, t) & \text { if }\left|u_{o}(x, t)-\bar{u}(x, t)\right| \leq k, \\
\bar{u}(x, t) & \text { if not, }\end{cases} \\
& \xi_{o k}(x, t)=\left\{\begin{array}{cl}
\xi_{o}(x, t) & \text { if }\left|\xi_{o}(x, t)-\bar{\xi}(x, t)\right| \leq k, \\
\bar{\xi}(x, t) & \text { if not. }
\end{array}\right.
\end{aligned}
$$

Observe that for every $k \geq 1,\left(u_{o k}, \xi_{o k}\right)$ belongs to $U_{a d}(\bar{u}, k) \times V_{a d}(\bar{\xi}, k)$, and that $\left(u_{o k}, \xi_{o k}\right)_{k}$ converges to $\left(u_{o}, \xi_{o}\right)$ in $L^{p}(Q) \times L^{p}(Q)$. Applying Theorem 4.1 of [7] (see also [24, 21] for more details), we deduce the existence of measurable sets $E_{\rho}^{k}$ with $\mathcal{L}^{n+1}\left(E_{\rho}^{k}\right)=\rho \mathcal{L}^{n+1}(Q)$, such that if we denote by $\left(u_{k}^{\rho}, \xi_{k}^{\rho}\right)$ the pair of controls defined by

$$
u_{k}^{\rho}(x, t)=\left\{\begin{array}{cc}
u_{k}(x, t) & \text { on } Q \backslash E_{\rho}^{k} \\
u_{o k}(x, t) & \text { on } E_{\rho}^{k}
\end{array}, \quad \xi_{k}^{\rho}(x, t)=\left\{\begin{array}{cc}
\xi_{k}(x, t) & \text { on } Q \backslash E_{\rho}^{k} \\
\xi_{\text {ok }}(x, t) & \text { on } E_{\rho}^{k}
\end{array}\right.\right.
$$

and if $y_{k}^{\rho}$ is the state corresponding to $\left(u_{k}^{\rho}, \xi_{k}^{\rho}\right)$, then we have

$$
\begin{gathered}
y_{k}^{\rho}=y_{k}+\rho z_{k}+r_{k}^{\rho}, \quad \lim _{\rho \rightarrow 0} \frac{1}{\rho}\left|r_{k}^{\rho}\right|_{\mathcal{C}(\bar{Q})}=0, \\
J\left(y_{k}^{\rho}, u_{k}^{\rho}\right)=J\left(y_{k}, u_{k}\right)+\rho \Delta_{k} J+o(\rho), \\
\int_{Q} y_{k}^{\rho} \xi_{k}^{\rho} d x d t=\int_{Q} y_{k} \xi_{k} d x d t+\rho \int_{Q}\left[z_{k} \xi_{k}+y_{k}\left(\xi_{o k}-\xi_{k}\right)\right] d x d t+o(\rho),
\end{gathered}
$$

where $z_{k}$ is the weak solution of

$$
\frac{\partial z_{k}}{\partial t}+A z_{k}+f_{y}^{\prime}\left(y_{k}\right) z_{k}=u_{k}-u_{o k}+\xi_{k}-\xi_{o k} \quad \text { in } Q, \quad z_{k}=0 \quad \text { on } \Sigma, \quad z_{k}(0)=0 \quad \text { in } \Omega,
$$

and

$$
\Delta_{k} J=\int_{Q}\left[F_{y}^{\prime}\left(x, t, y_{k}, u\right) z_{k}+F\left(x, t, y_{k}, u_{o k}\right)-F\left(x, t, y_{k}, u_{k}\right)\right] d x d t+\int_{\Omega} L_{y}^{\prime}\left(x, y_{k}(T)\right) z_{k}(T) d x .
$$

Setting $(u, \xi)=\left(u_{k}^{\rho}, \xi_{k}^{\rho}\right)$ in $(4.15 \mathrm{~b})$, it follows that

$$
\limsup _{\rho \rightarrow 0} \frac{J_{\varepsilon_{k}}\left(y_{k}, u_{k}, \xi_{k}\right)-J_{\varepsilon_{k}}\left(y_{k}^{\rho}, u_{k}^{\rho}, \xi_{k}^{\rho}\right)}{\rho} \leq \varepsilon_{k} \mathcal{L}^{n+1}(Q) .
$$

Taking (4.18) and the definition of $J_{\varepsilon_{k}}$ into account, we get

$$
-\nu_{k} \Delta_{k} J-\left\langle\mu_{k}, z_{k}\right\rangle_{\bar{Q}}-\left\langle\theta_{k}, \Phi^{\prime}\left(y_{k}\right) z_{k}\right\rangle_{\bar{Q}}-\lambda_{k}\left[\left\langle\xi_{k}, z_{k}\right\rangle_{\bar{Q}}+\left\langle y_{k}, \xi_{o k}-\xi_{k}\right\rangle_{\bar{Q}}\right] \leq \varepsilon_{k} \mathcal{L}^{n+1}(Q)
$$

where

$$
\nu_{k}=\frac{\left(J\left(y_{k}, u_{k}\right)-J(\bar{y}, \bar{u})+\varepsilon_{k}^{2}\right)^{+}}{J_{\varepsilon_{k}}\left(y_{k}, u_{k}, \xi_{k}\right)}, \lambda_{k}=\frac{\left(\int_{Q} y_{k}(x, t) \xi_{k}(x, t) d x d t\right)}{J_{\varepsilon_{k}}\left(y_{k}, u_{k}, \xi_{k}\right)},
$$




$$
\begin{gathered}
\mu_{k}= \begin{cases}\frac{\left|y_{k}^{-}\right|_{\mathcal{C}(\bar{Q})} \nabla\left|y_{k}^{-}\right|_{\mathcal{C}(\bar{Q})}}{J_{\varepsilon_{k}}\left(y_{k}, u_{k}, \xi_{k}\right)} & \text { if }\left|y_{k}^{-}\right|_{\mathcal{C}(\bar{Q})} \neq 0, \\
0 & \text { otherwise, }\end{cases} \\
\theta_{k}= \begin{cases}\frac{\delta_{\mathcal{C}}\left(\Phi\left(y_{k}\right) \nabla \delta_{\mathcal{C}}\left(\Phi\left(y_{k}\right)\right)\right.}{J_{\varepsilon_{k}}\left(y_{k}, u_{k}, \xi_{k}\right)} & \text { if } \delta_{\mathcal{C}}\left(\Phi\left(y_{k}\right)\right) \neq 0, \\
0 & \text { otherwise }\end{cases}
\end{gathered}
$$

For every $k>0$, we consider the weak solution $q_{k}$ of

$$
\left\{\begin{array}{c}
-\frac{\partial q_{k}}{\partial t}+A^{*} q_{k}+f_{y}^{\prime}\left(y_{k}\right) q_{k}=\nu_{k} F_{y}^{\prime}\left(x, t, y_{k}, u_{k}\right)+\left.\mu_{k}\right|_{Q}+\left.\left[\Phi^{\prime}\left(y_{k}\right)^{*} \theta_{k}\right]\right|_{Q}+\lambda_{k} \xi_{k} \quad \text { in } Q \\
q_{k}=0 \quad \text { on } \Sigma, \quad q_{k}(T)=\nu_{k} L_{y}^{\prime}\left(x, y_{k}(T)\right)+\left.\left[\Phi^{\prime}\left(y_{k}\right)^{*} \theta_{k}\right]\right|_{\bar{\Omega}_{T}}+\left.\mu_{k}\right|_{\bar{\Omega}_{T}} \quad \text { in } \Omega
\end{array}\right.
$$

where $\left.\mu_{k}\right|_{Q}$ (resp. $\left.\left.\left[\Phi^{\prime}\left(y_{k}\right)^{*} \theta_{k}\right]\right|_{Q}\right)$ is the restriction of $\mu_{k}$ (resp. $\left.\left[\Phi^{\prime}\left(y_{k}\right)^{*} \theta_{k}\right]\right)$ to $Q$, and $\left.\mu_{k}\right|_{\bar{\Omega}_{T}}$ (resp. $\left.\left.\left[\Phi^{\prime}\left(y_{k}\right)^{*} \theta_{k}\right]\right|_{\Omega_{T}}\right)$ is the restriction of $\mu_{k}$ (resp. $\left.\left[\Phi^{\prime}\left(y_{k}\right)^{*} \theta_{k}\right]\right)$ to $\bar{\Omega} \times\{T\}$. By using the Green formula of Proposition 4.1 with $z_{k}$, we obtain

$$
\begin{gathered}
\int_{Q} \nu_{k} F_{y}^{\prime}\left(x, t, y_{k}, u_{k}\right) z_{k} d x d t+\lambda_{k} \int_{Q} z_{k}(x, t) \xi_{k}(x, t) d x d t+\int_{\Omega} \nu_{k} L_{y}^{\prime}\left(x, y_{k}(T)\right) z_{k}(T) d x+ \\
\left\langle\mu_{k}, z_{k}\right\rangle_{\bar{Q}}+\left\langle\theta_{k}, \Phi^{\prime}\left(y_{k}\right) z_{k}\right\rangle_{\bar{Q}}=\int_{Q} q_{k}\left(u_{o k}-u_{k}+\xi_{o k}-\xi_{k}\right) d x d t .
\end{gathered}
$$

With this equality, (4.20) and the definition of $\Delta_{k} J$, we get

$$
\begin{aligned}
\int_{Q}\left[\nu_{k} F(x, t\right. & \left.\left., y_{k}, u_{k}\right)+q_{k} u_{k}+q_{k} \xi_{k}+\lambda_{k} y_{k} \xi_{k}\right] d s d t \\
& \leq \int_{Q}\left[\nu_{k} F\left(s, t, y_{k}, u_{o k}\right)+q_{k} u_{o k}+q_{k} \xi_{o k}+\lambda_{k} y_{k} \xi_{o k}\right] d s d t+\frac{1}{k^{2 p}} \mathcal{L}^{n+1}(Q)
\end{aligned}
$$

for every $k>0$ and every $\left(u_{o}, \xi_{o}\right) \in U_{a d} \times V_{a d}\left(\right.$ where $\left(u_{o k}, \xi_{o k}\right)$ is defined with respect to $\left.\left(u_{o}, \xi_{o}\right)\right)$.

Step 4. Convergence of sequence $\left(\nu_{k}, \lambda_{k}, \mu_{k}, \theta_{k}, q_{k}\right)_{k}$. Pontryagin principle

Observing that $\nu_{k}^{2}+\lambda_{k}^{2}+\left|\mu_{k}\right|_{\mathcal{M}(\bar{Q})}^{2}+\left|\theta_{k}\right|_{\mathcal{M}(\bar{Q})}^{2}=1$, there exist $(\bar{\nu}, \bar{\lambda}, \bar{\mu}, \bar{\theta}) \in \mathbb{R}^{+} \times \mathbb{R}^{+} \times \mathcal{M}(\bar{Q}) \times \mathcal{M}(\bar{Q})$ and a subsequence, still denoted by $\left(\nu_{k}, \lambda_{k}, \mu_{k}, \theta_{k}\right)_{k}$, such that

$$
\nu_{k} \rightarrow \bar{\nu}, \quad \lambda_{k} \rightarrow \bar{\lambda}, \quad \mu_{k} \rightarrow \bar{\mu} \text { and } \theta_{k} \rightarrow \bar{\theta} \text { weak }^{*} \text { in } \mathcal{M}(\bar{Q}) .
$$

With the same arguments as in ([21], Section 6.2 , Step 4), we prove that $\left(q_{k}\right)_{k}$, or at least a subsequence of $\left(q_{k}\right)_{k}$, weakly converges to $\bar{q}$ in $L^{\delta^{\prime}}\left(0, T ; W_{0}^{1, d^{\prime}}(\Omega)\right)$ for every $(\delta, d)$ such that $\frac{n}{2 d}+\frac{1}{\delta}<\frac{1}{2}$. Recall that $\left(u_{k}, \xi_{k}\right)_{k}$ converges to $(\bar{u}, \bar{\xi})$ in $L^{p}(Q) \times L^{p}(Q)$. Hence, $y_{k}$ also converges to $\bar{y}$. Passing to the limit when $k$ tends to infinity in (4.22) gives

$$
\int_{Q}\left[H_{1}(x, t, \bar{y}, \bar{u}, \bar{q}, \bar{\nu})+H_{2}(\bar{y}, \bar{\xi}, \bar{q}, \bar{\lambda})\right] d x d t \leq \int_{Q}\left[H_{1}(x, t, \bar{y}, u, \bar{q}, \bar{\nu})+H_{2}(\bar{y}, \xi, \bar{q}, \bar{\lambda})\right] d x d t,
$$

for every $(u, \xi) \in U_{a d} \times V_{a d}$. This inequality is equivalent to

$(4.23 \mathrm{a}) \int_{Q} H_{1}(x, t, \bar{y}(x, t), \bar{u}(x, t), \bar{q}(x, t), \bar{\nu}) d x d t=\min _{u \in U_{a d}} \int_{Q} H_{1}(x, t, \bar{y}(x, t), u(x, t), \bar{q}(x, t), \bar{\nu}) d x d t$ 


$$
\int_{Q} H_{2}(\bar{y}(x, t), \bar{\xi}(x, t), \bar{q}(x, t), \bar{\lambda}) d x d t=\min _{\xi \in V_{a d}} \int_{Q} H_{2}(\bar{y}(x, t), \xi(x, t), \bar{q}(x, t), \bar{\lambda}) d x d t .
$$

Now, by using Lebesgue's points argument (see [21, 24]), we obtain (4.10e) and (4.10f). On the other hand, it is clear that $\bar{\nu} \geq 0$. Moreover, from the definitions of $\mu_{k}$ and $\theta_{k}$, we deduce

$$
\left\langle\mu_{k}, z-y_{k}\right\rangle_{\bar{Q}} \leq 0 \quad \forall z \in\{z \in \mathcal{C}(\bar{Q}) \mid z \geq 0\}, \quad \text { and }\left\langle\theta_{k}, z-\Phi\left(y_{k}\right)\right\rangle_{\bar{Q}} \leq 0 \quad \forall z \in \mathcal{C} .
$$

When $k$ tends to infinity, we obtain (4.10b) and a part of (4.10a). It remains to prove that $(\bar{\nu}, \bar{\lambda}, \bar{\mu}, \bar{\theta})$ is nonzero; for this, we recall that $\nu_{k}^{2}+\lambda_{k}^{2}+\left|\mu_{k}\right|_{\mathcal{M}(\bar{Q})}^{2}+\left|\theta_{k}\right|_{\mathcal{M}(\bar{Q})}^{2}=1$.

If $(\bar{\nu}, \bar{\lambda}) \neq 0$, then the proof is complete. If not, we can prove that $|\bar{\mu}|_{\mathcal{M}(\bar{Q})}+|\bar{\theta}|_{\mathcal{M}(\bar{Q})}>0$.

First we recall that $\mathcal{C}$ has a finite codimension in $\mathcal{C}(\bar{Q})$ and that $\{z \in \mathcal{C}(\bar{Q}) \mid z \geq 0\}$ is a subset of $\mathcal{C}(\bar{Q})$ with a nonempty interior. Then $\widetilde{\mathcal{C}}$ is a subset of $\mathcal{C}(\bar{Q}) \times \mathcal{C}(\bar{Q})$ with a finite codimension. Moreover, from (4.24), we deduce that, for every $\left(z_{1}, z_{2}\right) \in \widetilde{\mathcal{C}}$ :

$\left\langle\mu_{k}, z_{2}-\bar{y}\right\rangle_{\bar{Q}}+\left\langle\theta_{k}, z_{1}-\Phi(\bar{y})\right\rangle_{\bar{Q}} \leq\left\langle\mu_{k}, y_{k}-\bar{y}\right\rangle_{\bar{Q}}+\left\langle\theta_{k}, \Phi\left(y_{k}\right)-\Phi(\bar{y})\right\rangle_{\bar{Q}} \leq\left|y_{k}-\bar{y}\right|_{\mathcal{C}(\bar{Q})}+\left|\Phi\left(y_{k}\right)-\Phi(\bar{y})\right|_{\mathcal{C}(\bar{Q})}$.

The last right-hand side quantity tends to 0 as $k \rightarrow+\infty$. With this estimate and using $\lim _{k}\left|\mu_{k}\right|_{\mathcal{M}(\bar{Q})}+$ $\lim _{k}\left|\theta_{k}\right|_{\mathcal{M}(\bar{Q})}=1$, thanks to Lemma 3.6 of [16], we conclude that $(\bar{\mu}, \bar{\theta}) \neq 0$ when $(\bar{\nu}, \bar{\lambda})=0$.

5. Examples . Let us consider the following optimal control problem where the cost functional is defined by

$$
J(y, u)=\int_{0}^{T}[g(t, y(t))+h(u(t))] d t+\psi(y(T))
$$

where

(A5*) the function $h: L^{2}(\Omega) \rightarrow \mathbb{R} \cup\{+\infty\}$ is convex and lower semicontinuous and there exist $c_{1}>$ $0, c_{2} \in \mathbb{R}$ such that

$$
\forall u \in L^{2}(\Omega) \quad h(u) \geq c_{1}|u|_{L^{2}(\Omega)}^{2}-c_{2}
$$

(A6 ${ }^{*}$ ) the function $g:[0, T] \times L^{2}(\Omega) \rightarrow \mathbb{R} \cup\{+\infty\}$ is measurable in $t, g(., 0) \in L^{1}(0, T)$, and for every $r>0$ there exists $\gamma_{r}>0$ independent of $t$ such that :

$$
\begin{gathered}
\forall t \in[0, T],|y|_{L^{2}(\Omega)}+|z|_{L^{2}(\Omega)} \leq r \\
|g(t, y)-g(t, z)|+|\psi(y)-\psi(z)| \leq \gamma_{r}|y-z|_{L^{2}(\Omega)} .
\end{gathered}
$$

Conditions on $g$ and $\psi$ could be weakened. For more details one can refer to Barbu [2] p.317.

Now we consider

$$
\left\{\begin{array}{l}
\text { Minimize } J\left(y\left(y_{o}, u\right), u\right) \\
u \in U_{a d}, \\
y\left(y_{o}, u\right) \text { is the solution of }(4.3) .
\end{array}\right.
$$

where $U_{a d}$ is a nonempty, convex subset of $L^{p}(Q)$, closed for the $L^{2}(Q)$-topology and $p$ is an integer such that $n<p$. Though we are especially interested in optimality conditions for solutions of problem $(\mathcal{P})$ we may give an existence result anyhow. More precisely :

Theorem 5.1. For any $y_{o} \in K_{o}$ (defined by (4.1)), problem $(\mathcal{P})$ has at least one solution u. Moreover the corresponding state belongs to $\mathcal{C}(\bar{Q}) \cap W^{2,1, p}(Q)$. 
Proof - One can find this result in Barbu [2] Proposition 1.1. p.319, when $U_{a d}=L^{2}(Q)$. It is easy to adapt it to the case where $U_{a d}$ is a closed convex subset of $L^{2}(Q)$. A priori estimations do not change so that we get the "suitable" convergence in the "suitable" spaces. The only modification concerns the cluster points of the control sequences. As $U_{a d}$ is convex and closed for the $L^{2}(Q)$-topology these points belong to $U_{a d}$. As $U_{a d} \subset L^{p}(Q)$ we can use regularity results of Theorem 4.1.

REMARK 5.1. The assumption that $U_{\text {ad }}$ has to be a convex subset of $L^{p}(Q)$ (for some $p>n$ ) closed for the $L^{2}(Q)$-topology, may be difficult to ensure: for example $U_{a d}=L^{p}(Q)$ is not suitable. However, we give more precise example sets $U_{\text {ad }}$ in the sequel. Let us precise a little more the example; we set

$$
J(y, u)=\frac{1}{2} \int_{\Omega}\left(y(x, T)-z_{d}(x)\right)^{2} d x+\frac{N}{2} \int_{Q} u(x, t)^{2} d x d t
$$

(with $N>0$ ), so that with the previous notations we get

$$
\begin{gathered}
F(x, t, y, u)=\frac{N}{2} u^{2}, h(u(t))=\frac{N}{2}\|u(t)\|_{L^{2}(\Omega)}^{2}, \\
L(x, y)=\frac{1}{2}\left(y-z_{d}(x)\right)^{2}, g(t, y(t)) \equiv 0, \psi(y(T))=\frac{1}{2}\left\|y(T)-z_{d}\right\|_{L^{2}(\Omega)}^{2} .
\end{gathered}
$$

It is easy to see that both $\left(\mathrm{A} 5^{*}\right)$ and $\left(\mathrm{A} 6^{*}\right)$ are fulfilled for such a choice of $h, g, \psi$. Therefore the following optimal control problem

$$
\left\{\begin{array}{l}
\min J(y, u) \\
\frac{\partial y}{\partial t}+A y+f(y) \geq u \text { in } Q, \quad y=0 \text { on } \Sigma, \quad y(0)=y_{o} \text { in } \Omega \\
u \in U_{a d}, \\
y(x, t) \geq 0 \forall(x, t) \in \bar{Q},
\end{array}\right.
$$

with $y_{o} \in W_{o}^{1, p}(\Omega), y_{o} \geq 0, z_{d} \in L^{2}(\Omega)$ and $U_{a d}$ a nonempty,convex subset of $L^{p}(Q)$ closed for the $L^{2}(Q)$-topology has an optimal solution.

We always assume of course (A1)-(A2) (one may choose $A=-\Delta$ for instance, where $\Delta$ is the laplacian operator); we have already seen that (A3) and (A4) are fulfilled with the special choice of $\varphi$ and $y_{0}$. It is also easy to see that (A5) and (A6) are ensured with $F$ and $L$ defined as above. So we may give optimality conditions for $\left(\mathcal{P}_{2}\right)$

Theorem 5.2. Assume (A1) and (A2). Then problem $\left(\mathcal{P}_{2}\right)$ has an optimal solution $(\bar{y}, \bar{u}) \in$ $\left[W^{2,1, p}(Q) \cap \mathcal{C}(\bar{Q})\right] \times L^{p}(Q)$. Moreover, there exist $(\bar{\nu}, \bar{\lambda}, \bar{\mu}, \bar{q}) \in \mathbb{R} \times \mathbb{R} \times \mathcal{M}(\bar{Q}) \times L^{1}\left(0, T ; W_{o}^{1,1}(\Omega)\right)$ such that the following optimality system holds

$$
\begin{gathered}
(\bar{\nu}, \bar{\lambda}, \bar{\mu}) \neq 0, \quad \bar{\nu} \geq 0, \\
\forall z \in\{z \in \mathcal{C}(\bar{Q}) \mid z \geq 0\} \quad\langle\bar{\mu}, z-\bar{y}\rangle_{\bar{Q}} \leq 0, \\
\left\{\begin{array}{c}
\frac{\partial \bar{y}}{\partial t}+A \bar{y}+f(\bar{y})=\bar{u}+\bar{\xi} \quad \text { in } Q, \\
\bar{y}=0 \quad \text { on } \Sigma, \quad \bar{y}(0)=y_{o} \quad \text { in } \Omega,
\end{array}\right. \\
\bar{y} \geq 0, \bar{\xi} \in V_{a d}, \quad \bar{u} \in U_{a d}, \int_{\Omega} \bar{y}(t) \bar{\xi}(t) d x=0 \quad \text { a.e. on }[0, T],
\end{gathered}
$$




$$
\begin{gathered}
\left\{\begin{array}{c}
-\frac{\partial \bar{q}}{\partial t}+A^{*} \bar{q}+f^{\prime}(\bar{y}) \bar{q}=\bar{\mu}_{\mid Q}+\bar{\lambda} \bar{\xi} \quad \text { in } Q, \\
\bar{q}=0 \quad \text { on } \Sigma, \quad \bar{q}(T)=\bar{\nu}\left[\bar{y}(T)-z_{d}\right]+\bar{\mu}_{\mid \bar{\Omega}_{T}} \quad \text { in } \Omega,
\end{array}\right. \\
[(\bar{\nu} N \bar{u}+\bar{q})(u-\bar{u})](x, t)) \leq 0 \quad \text { for all } u \in U_{a d}, \quad \text { and a.e. }(x, t) \in Q, \\
\bar{q}(x, t) \bar{\xi}(x, t)=0 \quad \text { a.e. }(x, t) \in Q,
\end{gathered}
$$

where $\bar{\xi}=\frac{\partial \bar{y}}{\partial t}+A \bar{y}+f(\bar{y})-\bar{u}$.

Proof - It is a direct consequence of Theorem 4.2 with $\Phi=I d$ and $\mathcal{C}$ is the whole space. Considering the Hamiltonian functions and relations (4.10e) and (4.10f) gives (5.5e) and (5.5f) immediately.

We end this section with two examples for $U_{a d}$

5.1. Case where $U_{a d}$ is bounded in $L^{\infty}(Q)$. Let us set

$$
U_{a d}=\left\{u \in L^{\infty}(Q) \mid a(x, t) \leq u(x, t) \leq b(x, t) \text { in } Q\right\},
$$

where $a, b \in L^{\infty}(Q) . U_{a d}$ is of course a convex subset of $L^{p}(Q)$ for any $p>n$. Moreover, we get

LEMma 5.1. $U_{a d}$ is closed for the $L^{2}(Q)$-topology.

Proof - let $u_{n} \in U_{a d}$ converging to $u$ in $L^{2}(Q)$. Then $u_{n}(x, t)$ converges to $u(x, t)$ almost everywhere in $Q$ so that we get $a(x, t) \leq u(x, t) \leq b(x, t)$ almost everywhere in $Q$. So $u \in L^{\infty}(Q)$. It is clear that $u \in U_{a d}$.

Therefore, in view of Remark 5.1 we get the following result for $y_{o}=0$ and

$$
J(y, u)=\frac{1}{2} \int_{\Omega}\left(y(x, T)-z_{d}(x)\right)^{2} d x+\frac{N}{2} \int_{Q} u^{2}(x, t) d x d t
$$

TheOrem 5.3. Assume (A1) and (A2). Then problem $\left(\mathcal{P}_{2}\right)$ has an optimal solution $(\bar{y}, \bar{u}) \in$ $\left[W^{2,1, p}(Q) \cap \mathcal{C}(\bar{Q})\right] \times L^{p}(Q)$, for any $p>n$. Moreover, there exists $(\bar{\nu}, \bar{\lambda}, \bar{\mu}, \bar{q}) \in \mathbb{R} \times \mathbb{R} \times \mathcal{M}(\bar{Q}) \times$ $L^{1}\left(0, T ; W_{o}^{1,1}(\Omega)\right)$ such that $(5.5 a)-(5.5 d)$ and $(5.5 g)$ hold with

$$
\begin{gathered}
\left\{\begin{array}{c}
-\frac{\partial \bar{q}}{\partial t}+A^{*} \bar{q}+f^{\prime}(\bar{y}) \bar{q}=\bar{\mu}_{\mid Q}+\bar{\lambda} \bar{\xi} \quad \text { in } Q, \\
\bar{q}=0 \quad \text { on } \Sigma, \quad \bar{q}(T)=\bar{\nu}\left[\bar{y}(T)-z_{d}\right]+\bar{\mu}_{\left.\right|_{\Omega_{T}}} \quad \text { in } \Omega,
\end{array}\right. \\
[(\bar{\nu} N \bar{u}+\bar{q})(u-\bar{u})](x, t)) \leq 0 \quad \text { for all } u \in U_{a d}, \quad \text { and a.e. }(x, t) \in Q .
\end{gathered}
$$

5.2. Case where $U_{a d}=\left\{u \in L^{p}(Q) \mid u(x, t) \geq 0\right.$ a.e. in $\left.Q\right\}$. When $U_{a d}=\left\{u \in L^{p}(Q) \mid u(x, t) \geq 0\right.$ a.e. in $Q\}$ and $y_{o} \geq 0$ in $\Omega$, thanks to the maximum principle for parabolic equations the constraint $y \geq 0$ is automatically fulfilled in equation (4.6b) so that the corresponding multiplier $\bar{\mu}$ is equal to 0 (or at least does not appear.) So the corresponding Pontryagin optimality system consists in equations $(5.5 \mathrm{a}),(5.5 \mathrm{c})-(5.5 \mathrm{~g})$ where equation $(5.5 \mathrm{e})$ is replaced by

$$
\left\{\begin{array}{c}
-\frac{\partial \bar{q}}{\partial t}+A^{*} \bar{q}+f^{\prime}(\bar{y}) \bar{q}=\bar{\lambda} \bar{\xi} \quad \text { in } Q \\
\bar{q}=0 \quad \text { on } \Sigma, \quad \bar{q}(T)=\bar{\nu}\left[\bar{y}(T)-z_{d}\right] \quad \text { in } \Omega
\end{array}\right.
$$

This implies in particular that $\bar{q} \in W^{2,1, p}(Q) \cap \mathcal{C}(\bar{Q})$.

For this simple example, we can see that the optimality conditions (5.2) are not trivial, because we cannot have $\bar{\nu}=\bar{\lambda}=0$. 
6. Conclusion. The optimality conditions we have obtained are given in a non qualified form. So far it is difficult to compare precisely these results to those already existing, since they are most of time in a qualified form $[6,5,17]$ or concern elliptic variational inequalities. Nevertheless we must underline that in this paper, we obtain interesting informations about optimal solutions (at least in simple cases) . Indeed, we have seen in Example 5 that the equation (5.5e) provides a quite precise information on the structure of the multipliers $\bar{\mu}+\bar{\xi} \bar{\lambda}$ for the distributed multiplier for instance and the adjoint state $\bar{q}$ : the regular part of this adjoint state belongs to $\mathcal{C}(\bar{Q})$ while the non-smooth part belongs to $L^{1}\left(0, T ; W_{o}^{1,1}(\Omega)\right)$. These informations seem new (to compare with those given in Barbu [2], Section 5.1.4 p 331, for example).

The method developed in $[5,23]$ for elliptic variational inequalities, is still true for the parabolic case, but we think that this method does not allow to obtain the condition (4.11b). However, in [23,5], the authors give a qualification assumption under which they can derive Pontryagin's principle in qualified form.

As we can preview now the generic form of the Lagrange multipliers, one can check optimal control problems where the variational inequality is more general than the obstacle type one or occurs on the boundary, with boundary control.

\section{REFERENCES}

[1] V. Barbu -Th. Precupanu, Convexity and optimization in Banach spaces, Sijthoff and Noordhoff, Leyden, the Netherlands (1978).

[2] V. Barbu, Analysis and Control of Non Linear Infinite Dimensional Systems, Math in Science and Engineering, Vol. 190, Academic Press 1993.

[3] M. Bergounioux, Optimal control of abstract elliptic variational inequalities with state constraints, SIAM Journal on Control and Optimization, Vol. 36, n 1 , pp.273-289, 1998.

[4] M. Bergounioux - F. Tröltzsch, Optimality Conditions and Generalized Bang-Bang Principle for a State Constrained Semilinear Parabolic Problem, Numerical Functional Analysis and Optimization, Vol. 15, no 5\&6, pp.517-537, 1996.

[5] J. F. Bonnans - E. Casas, An Extension of Pontryagin's Principle for State Constrained Optimal Control of Semilinear Elliptic Equations and Variational Inequalities, SIAM Journal on Control and Optimization, Vol. 33, 1995.

[6] J. F. Bonnans - D. Tiba, Pontryagin's Principle in the Control of Semilinear Elliptic Variational Inequalities, Applied Mathematics and Optimization, Vol. 23, pp. 299-312, 1991.

[7] E. Casas - J.-P. Raymond - H. Zidani, Control Problems for Parabolic Equations with Integral Controls-State constraints, preprint.

[8] F. H. Clarke, Optimization and Nonsmooth Analysis, John Wiley, New York, 1983.

[9] E. DiBenedetto, Degenerate Parabolic Equations, Springer-Verlag, New York, 1993.

[10] E. Di Benedetto, On the local behavior of solutions of degenerate parabolic equations with measurable coefficients, Annali della Scuola Normale Superiore di Pisa, Ser. I, Vol 13, nº 3, pp. 487-535, 1986.

[11] J. Diestel, Geometry of Banach Spaces- Selected Topics, Lecture Notes in Mathematics No 485, SpringerVerlag/Berlin/Heidelberg/New York, 1975.

[12] I. Ekeland, On the Variational Principle, Journal of Mathematical Analysis and Applications, Vol. 47, p. 324-353, 1974.

[13] I. Ekeland - R. Temam, Analyse convexe et problèmes variationnels, Dunod-Gauthier-Villars, Paris, 1974.

[14] H. O. Fattorini - S. Sritharan, Necessary and Sufficient Conditions for Optimal Controls in Viscous Flow Problems, Proceedings of the Royal Society of Edinburgh, 124A, p. 211-251, 1994.

[15] O. A. Ladyzenskaja - V. A. Solonnikov - N. N. Ural'ceva, Linear and Quasilinear Equations of Parabolic Type , Translations of Mathematical Monographs, Vol. 23, American Mathematical Society, Providence, R. I., 1968.

[16] X.-J. Li - J. Yong, Optimal Control Theory for Infinite Dimensional Systems, Birkhäuser/Boston, 1995. 
[17] F. Mignot - J.-P. Puel, Optimal Control in Some Variational Inequalities, Math. Cont. Theo., Vol. 14 (1985), 409-422.

[18] M. K. V. Murty - G. Stampacchia, A Variational Inequality with Mixed Boundary Conditions, Israel Journal of Mathematics, Vol. 13, 188-224, 1972.

[19] J.P. Raymond, Nonlinear Boundary Control of Semilinear Parabolic Problems with Pointwise State Constraints , Discontinuous and Continuous Dynamical Systems, Vol. 9, 341-370, 1997.

[20] J.P. Raymond - H. Zidani, Hamiltonian Pontryagins Principles for Control Problems Governed by Semilinear Parabolic Equations, to appear in Applied Mathematical and Optimization.

[21] J. P. Raymond - H. Zidani, Pontryagin's Principles for State-Constrained Control Problems Governed by Semilinear Parabolic Equations with unbounded Controls, to appear in SIAM Journal on Control and Optimization.

[22] D. Tiba, Optimal control of nonsmooth distributed parameter systems, Lecture Notes in Mathematics $\mathrm{n}^{\mathrm{O}} 1459$, Springer-Verlag, Berlin, 1990.

[23] J. Yong, Pontryagin Maximum Principle for Semilinear Second Order Elliptic Partial Differential Equations and Variational Inequalities with State Constraints, Differential and Integral Equations, Vol. 5, 1307-1334, 1992.

[24] H. Zidani, Optimal Control Problem for Semilinear Parabolic Equations: Optimality Conditions and Numerical Approximations, Thèse de doctorat, Université Paul Sabatier, Toulouse 1996. 